\title{
A nonlocal singular boundary value problem for second-order differential equations
}

Svatoslav Stanĕk 


\title{
A NONLOCAL SINGULAR BOUNDARY VALUE PROBLEM FOR SECOND-ORDER DIFFERENTIAL EQUATIONS
}

\author{
SVATOSLAV STANĚK
}

\begin{abstract}
We discuss the singular differential equation $\left(g\left(x^{\prime}\right)\right)^{\prime}=f\left(t, x, x^{\prime}\right)$ together with the nonlocal boundary conditions $\max \{x(t): t \in[0, T]\}=A, x(0)=$ $x(T)$. Here, $g \in C^{0}(\mathbb{R})$ is an increasing and odd function, whereas the negative $f$ satisfying the local Carathéodory conditions on $[0, T] \times \mathbb{R} \times(\mathbb{R} \backslash\{0\})$ may be singular at the value 0 of its second phase variable and $A \in \mathbb{R}$. An existence result for the above boundary value problem is proved by the regularization and sequential techniques. The proofs use the Leray-Schauder degree principle and the Vitali convergence theorem.
\end{abstract}

Mathematics Subject Classification: 34B16, 34B15

Keywords: Singular boundary value problem, singular second-order differential equation, nonlocal boundary condition, Leray-Schauder degree, Vitali convergence theorem

\section{InTRODUCtion}

Et $T$ be a positive number, $J=[0, T]$, and let $A \in \mathbb{R}$. Consider the boundary value problem

$$
\begin{gathered}
\left(g\left(x^{\prime}(t)\right)\right)^{\prime}=f\left(t, x(t), x^{\prime}(t)\right), \\
x(0)=x(T), \quad \max \{x(t): t \in J\}=A .
\end{gathered}
$$

Here, $g \in C^{0}(\mathbb{R})$ is an increasing and odd function, and the function $f$ satisfying local Carathéodory conditions on $J \times \mathbb{R} \times(\mathbb{R} \backslash\{0\})(f \in \operatorname{Car}(J \times \mathbb{R} \times(\mathbb{R} \backslash\{0\})))$ is negative, and it may be singular at the value 0 of its second phase variable in the following sense: $\lim _{y \rightarrow 0} f(t, x, y)=-\infty$ for a. e. $t \in J$ and every $x \in \mathbb{R}$.

We say that a function $x \in C^{1}(J)$ is a solution of the boundary value problem (1.1), (1.2) if $g\left(x^{\prime}\right) \in A C(J)$ (absolutely continuous functions on $J$ ), $x$ satisfies the boundary conditions (1.2), and (1.1) holds a. e. on $J$.

The aim of this paper is to give conditions guaranteeing the solvability of the boundary value problem (1.1), (1.2). We note that the derivative of any solution of the boundary value problem (1.1), (1.2) 'goes through' the singularity of $f$ somewhere

Supported by Grant No. 201/01/1451 of the Grant Agency of the Czech Republic and by the Council of the Czech Government J14/98:153100011. 
inside of $J$. Moreover, we consider solutions $x$ of the boundary value problem (1.1), (1.2) having the 'maximal' smoothness, that is, $x \in C^{1}(J)$ and $g\left(x^{\prime}\right) \in A C(J)$. Till the present, various types of nonlocal boundary conditions have been considered for differential equations, systems of differential equations, and systems of functional differential equations with continuous or Carathéodory nonlinearities (see, e. g., [4$6,8-10,13,16-18]$ and references therein) or nonlinearities having singularities in the time variable $t$ (see, e. g., $[12,14,15]$ and references therein). There are only a few papers (see $[1,2,19,20]$ ) dealing with boundary value problems for nonlocal boundary conditions and second-order differential equations having singularities in phase variables.

As usual, $\|x\|=\max \{|x(t)|: t \in J\}$ and $\|x\|_{L}=\int_{0}^{T}|x(t)| d t$ stand for the norm in $C^{1}(J)$ and $L_{1}(J)$, respectively. For every measurable set $\mathcal{M} \subset \mathbb{R}$, its Lebesgue measure is denoted by the symbol $\mu(\mathcal{M})$.

Throughout the paper, the following assumptions are used.

$\left(H_{1}\right) g \in C^{0}(\mathbb{R})$ is an increasing and odd function, $\lim _{u \rightarrow \infty} g(u)=\infty$;

$\left(H_{2}\right) f \in \operatorname{Car}(J \times \mathbb{R} \times(\mathbb{R} \backslash\{0\}))$ and there exists a positive constant $a$ such that

$$
f(t, x, y) \leq-a
$$

for a. e. $t \in J$ and all $(x, y) \in \mathbb{R} \times(\mathbb{R} \backslash\{0\})$;

$\left(H_{3}\right)$ For a. e. $t \in J$ and all $(x, y) \in \mathbb{R} \times(\mathbb{R} \backslash\{0\})$,

$$
f(t, x, y) \geq-q(x)\left(\omega_{1}(|y|)+\omega_{2}(|y|)\right)
$$

with $q \in C^{0}(\mathbb{R})$ positive, $\omega_{1} \in C^{0}([0, \infty))$ non-negative, $\omega_{2} \in C^{0}((0, \infty))$ positive and non-increasing and

$$
\int_{0}^{1} \omega_{2}\left(g^{-1}(s)\right) d s<\infty \text {. }^{*}
$$

A further assumption $\left(H_{4}\right)$ will be given in Section 2.

The plan of the paper is as follows: In Section 2, we first define a sequence of auxiliary regular boundary value problems with nonlinearities $f_{n}$ such that $f_{n}(t, x, y)=$ $f(t, x, y)$ for a. e. $t \in J$ and for $x \in \mathbb{R},|y| \geq 1 / n, n \in \mathbb{N}$. Then we consider properties of their solutions (Lemma 2.1) and give them a priori bounds (Lemma 2.2). By using the Leray-Schauder degree principle (see [7]), the existence of a solution $x_{n}$ of auxiliary boundary value problem with nonlinearity $f_{n}$ is proved (Lemma 2.3). Finally, we show that the sequence $\left\{f_{n}\left(t, x_{n}(t), x_{n}^{\prime}(t)\right)\right\}$ is uniformly absolutely continuous on $J$ (Lemma 2.5). It is this result which is used in the next section when passing to the limit because it is impossible to find a Lebesque majorant for $\left\{f_{n}\left(t, x_{n}(t), x_{n}^{\prime}(t)\right)\right\}$ which is necessary for the application of the Lebesgue theorem on dominated convergence.

* Since $g^{-1}$ is increasing by $\left(H_{1}\right)$ and $\omega_{2}$ is positive and non-increasing by $\left(H_{3}\right)$, it follows that $\int_{0}^{c} \omega_{2}\left(g^{-1}(s)\right) d s<\infty$ for every $c \in(0, \infty)$. 
Section 3 presents the main existence result for singular boundary value problem (1.1), (1.2) (Theorem 3.1). It is proved by the Arzelà-Ascoli theorem and the Vitali convergence theorem (see $[3,11])$.

The results are demonstrated on an example (Example 3.2).

\section{Auxiliary Regular boundary value problems}

For every $n \in \mathbb{N}$, we define $f_{n} \in \operatorname{Car}\left(J \times \mathbb{R}^{2}\right)$ by

$$
f_{n}(t, x, y)= \begin{cases}f(t, x, y) & \text { for }(t, x) \in J \times \mathbb{R},|y| \geq \frac{1}{n} \\ \frac{n}{2}\left[f\left(t, x, \frac{1}{n}\right)\left(y+\frac{1}{n}\right)-f\left(t, x,-\frac{1}{n}\right)\left(y-\frac{1}{n}\right)\right] & \text { for }(t, x) \in J \times \mathbb{R},|y|<\frac{1}{n} .\end{cases}
$$

By $\left(H_{2}\right)$ and $\left(H_{3}\right)$,

$$
-a \geq f_{n}(t, x, y) \geq-q(x)\left(\omega_{* 1}(|y|)+\omega_{2}(|y|)\right)
$$

for a. e. $t \in J$ and all $(x, y) \in \mathbb{R}^{2}$, where

$$
\omega_{* 1}(x)= \begin{cases}\omega_{1}(1) & \text { for } 0 \leq x \leq 1 \\ \omega_{1}(x) & \text { for } x>1\end{cases}
$$

Consider the family of regular differential equations

$$
\left(g\left(x^{\prime}(t)\right)\right)^{\prime}=\lambda f_{n}\left(t, x(t), x^{\prime}(t)\right)
$$

depending on the parameters $n \in \mathbb{N}$ and $\lambda \in[0,1]$. We note that $x=A$ is the unique solution of the boundary value problem $(\mathrm{E})_{n}^{0},(1.2)$ for every $n \in \mathbb{N}$.

Lemma 2.1. Let assumptions $\left(H_{1}\right)-\left(H_{3}\right)$ be satisfied and let $n \in \mathbb{N}, \lambda \in(0,1]$. Assume that $x$ is a solution of the boundary value problem $(\mathrm{E})_{n}^{\lambda},(1.2)$. Then

$$
\begin{gathered}
(x(T)=) x(0)=\min \{x(t): t \in J\}<A, \\
A-x(0) \geq \int_{0}^{T / 2} g^{-1}(\lambda a s) d s,
\end{gathered}
$$

$x^{\prime}(\xi)=0$ for a unique $\xi \in(0, T)$, and

$$
\begin{aligned}
& x^{\prime}(t) \geq g^{-1}(\lambda a(\xi-t)) \quad \text { for } t \in[0, \xi], \\
& x^{\prime}(t) \leq-g^{-1}(\lambda a(t-\xi)) \quad \text { for } t \in[\xi, T] .
\end{aligned}
$$

Proof. Since

$$
\left(g\left(x^{\prime}(t)\right)\right)^{\prime} \leq-\lambda a \text { for a. e. } t \in J,
$$

by (2.1), we see that $g\left(x^{\prime}\right)$ is decreasing on $J$ and, therefore, $x^{\prime}$ is decreasing as well. Now from (1.2) we deduce that $x^{\prime}(\xi)=0$ for a $\xi \in(0, T)$, and so $\xi$ is unique, $\max \{x(t): t \in J\}=x(\xi), x^{\prime}>0$ on $[0, \xi) x^{\prime}<0$ on $(\xi, T]$, and (2.2) is also true. Integrating (2.5) over $[t, \xi] \subset[0, \xi]$ and over $[\xi, t] \subset[\xi, T]$, we get

$$
g\left(x^{\prime}(t)\right) \geq \lambda a(\xi-t), \quad t \in[0, \xi]
$$


and

$$
g\left(x^{\prime}(t)\right) \leq-\lambda a(t-\xi), \quad t \in[\xi, T],
$$

respectively. Hence, (2.4) is true.

Finally, it follows from (2.4) and the equality $x(\xi)=A$ that

$$
A-x(0)=x(\xi)-x(0)=\int_{0}^{\xi} x^{\prime}(t) d t \geq \int_{0}^{\xi} g^{-1}(\lambda a(\xi-t)) d t=\int_{0}^{\xi} g^{-1}(\lambda a s) d s
$$

and

$$
\begin{aligned}
x(T)-A & =x(T)-x(\xi)=\int_{\xi}^{T} x^{\prime}(t) d t \leq-\int_{\xi}^{T} g^{-1}(\lambda a(t-\xi)) d t \\
& =-\int_{0}^{T-\xi} g^{-1}(\lambda a s) d s .
\end{aligned}
$$

Therefore,

$$
\begin{aligned}
(A-x(T)=) A-x(0) & \geq \max \left\{\int_{0}^{\xi} g^{-1}(\lambda a s) d s, \int_{0}^{T-\xi} g^{-1}(\lambda a s) d s\right\} \\
& \geq \int_{0}^{T / 2} g^{-1}(\lambda a s) d s,
\end{aligned}
$$

which proves (2.3).

We are going to give a priori bounds for the solution of the boundary value problem $(\mathrm{E})_{n}^{\lambda},(1.2)$. Define the functions $H \in C^{0}([0, \infty))$ and $Q_{A} \in C^{0}((-\infty, A]), A \in \mathbb{R}$, by the relations

$$
H(u)=\int_{0}^{g(u)} \frac{g^{-1}(s)}{\omega_{* 1}\left(g^{-1}(s)\right)+\omega_{2}\left(g^{-1}(s)\right)} d s
$$

and

$$
Q_{A}(u)=\int_{u}^{A} q(s) d s
$$

Then $H$ is increasing on $[0, \infty), Q_{A}$ is decreasing on $(-\infty, A]$, and $H^{-1}\left(Q_{A}(A)\right)=0$. If the function $H^{-1}\left(Q_{A}(u)\right)$ satisfies the assumption ${ }^{\dagger}$

$\left(H_{4}\right)$

$$
\int_{A-1}^{A} \frac{1}{H^{-1}\left(Q_{A}(s)\right)} d s<\infty \quad \text { for } A \in \mathbb{R}
$$

then we define, for every $A \in \mathbb{R}$, the function $S_{A} \in C^{0}((-\infty, A])$ by putting

$$
S_{A}(u)=\int_{u}^{A} \frac{1}{H^{-1}\left(Q_{A}(s)\right)} d s .
$$

\footnotetext{
${ }^{\dagger}$ Since the function $H^{-1}\left(Q_{A}\right)$ is decreasing on $(-\infty, A]$, the assumption $\left(H_{4}\right)$ guarantees that $\int_{u}^{A} \frac{d s}{H^{-1}\left(Q_{A}(s)\right)}<\infty$ for every $u \in(-\infty, A)$.
} 
Then $S_{A}(A)=0$ and $S_{A}$ is decreasing on $(-\infty, A]$. Set

$$
\mathcal{A}=\left\{A: A \in \mathbb{R}, \lim _{u \rightarrow-\infty} S_{A}(u)>\frac{T}{2}\right\} .
$$

We are now in a position to give a priori bounds for the solution of the boundary value problem $(\mathrm{E})_{n}^{\lambda},(1.2)$.

Lemma 2.2. Let assumptions $\left(H_{1}\right)-\left(H_{4}\right)$ be satisfied and let $A \in \mathcal{A}$. Let $x$ be a solution of the boundary value problem $(\mathrm{E})_{n}^{\lambda},(1.2), \lambda \in(0,1], n \in \mathbb{N}$. Then

$$
x(0)=x(T) \geq \Lambda_{A}
$$

and

where

$$
\|x\| \leq \max \left\{\left|\Lambda_{A}\right|,|A|\right\}, \quad\left\|x^{\prime}\right\| \leq H^{-1}\left(Q_{A}\left(\Lambda_{A}\right)\right),
$$

and $S_{A}$ is defined by (2.6).

$$
\Lambda_{A}=S_{A}^{-1}\left(\frac{T}{2}\right)
$$

Proof. By Lemma 2.1, $x^{\prime}(\xi)=0$ for a unique $\xi \in(0, T), x^{\prime}>0$ on $[0, \xi)$, and $x^{\prime}<0$ on $(\xi, T]$. Then (see $(2.1))$

$$
\left(g\left(x^{\prime}(t)\right)\right)^{\prime} x^{\prime}(t) \geq-q(x(t))\left[\omega_{* 1}\left(x^{\prime}(t)\right)+\omega_{2}\left(x^{\prime}(t)\right)\right] x^{\prime}(t) \quad \text { for a. e. } t \in[0, \xi]
$$

and

$$
\left(g\left(x^{\prime}(t)\right)\right)^{\prime} x^{\prime}(t) \leq-q(x(t))\left[\omega_{* 1}\left(-x^{\prime}(t)\right)+\omega_{2}\left(-x^{\prime}(t)\right)\right] x^{\prime}(t) \quad \text { for a. e. } t \in[\xi, T] .
$$

Integrating the inequality

$$
\frac{\left(g\left(x^{\prime}(t)\right)\right)^{\prime} x^{\prime}(t)}{\omega_{* 1}\left(x^{\prime}(t)\right)+\omega_{2}\left(x^{\prime}(t)\right)} \geq-q(x(t)) x^{\prime}(t) \quad \text { for a. e. } t \in[0, \xi]
$$

from $t \in[0, \xi]$ to $\xi$ and the inequality

$$
\frac{\left(g\left(x^{\prime}(t)\right)\right)^{\prime} x^{\prime}(t)}{\omega_{* 1}\left(-x^{\prime}(t)\right)+\omega_{2}\left(-x^{\prime}(t)\right)} \leq-q(x(t)) x^{\prime}(t) \quad \text { for a. e. } t \in[\xi, T]
$$

from $\xi$ to $t \in[\xi, T]$ and using the relation $x(\xi)=A$, we get

$$
\int_{0}^{g\left(x^{\prime}(t)\right)} \frac{g^{-1}(s)}{\omega_{* 1}\left(g^{-1}(s)\right)+\omega_{2}\left(g^{-1}(s)\right)} d s \leq \int_{x(t)}^{A} q(s) d s, \quad t \in[0, \xi]
$$

and

Therefore,

$$
\int_{0}^{g\left(-x^{\prime}(t)\right)} \frac{g^{-1}(s)}{\omega_{* 1}\left(g^{-1}(s)\right)+\omega_{2}\left(g^{-1}(s)\right)} d s \leq \int_{x(t)}^{A} q(s) d s, \quad t \in[\xi, T] .
$$

and

$$
H\left(x^{\prime}(t)\right) \leq Q_{A}(x(t)) \text { for } t \in[0, \xi]
$$

$$
H\left(-x^{\prime}(t)\right) \leq Q_{A}(x(t)) \quad \text { for } t \in[\xi, T] .
$$


Now integrating the relation

from 0 to $\xi$ and

$$
\frac{x^{\prime}(t)}{H^{-1}\left(Q_{A}(x(t))\right.} \leq 1, \quad t \in[0, \xi]
$$

$$
-\frac{x^{\prime}(t)}{H^{-1}\left(Q_{A}(x(t))\right.} \leq 1, \quad t \in[\xi, T]
$$

from $\xi$ to $T$, we have (see (2.6)) $S_{A}(x(0)) \leq \xi$ and $S_{A}(x(T)) \leq T-\xi$. Hence $\left(S_{A}(x(T))=\right) S_{A}(x(0)) \leq T / 2$, and consequently $(x(T)=) x(0) \geq S_{A}^{-1}(T / 2)=\Lambda_{A}$ because $S_{A}$ is decreasing. We have thus proved that (2.8) is true and, therefore,

$$
\|x\| \leq \max \left\{\left|\Lambda_{A}\right|,|A|\right\} .
$$

Now (2.11) with $t=0$ and (2.12) with $t=T$ yield

$$
\begin{gathered}
x^{\prime}(0) \leq H^{-1}\left(Q_{A}(x(0)) \leq H^{-1}\left(Q_{A}\left(\Lambda_{A}\right)\right),\right. \\
-x^{\prime}(T) \leq H^{-1}\left(Q_{A}(x(T)) \leq H^{-1}\left(Q_{A}\left(\Lambda_{A}\right)\right),\right.
\end{gathered}
$$

and since $\left\|x^{\prime}\right\|=\max \left\{x^{\prime}(0),-x^{\prime}(T)\right\}$, we have

$$
\left\|x^{\prime}\right\| \leq H^{-1}\left(Q_{A}\left(\Lambda_{A}\right)\right)
$$

as required.

Lemma 2.3. Let assumptions $\left(H_{1}\right)-\left(H_{4}\right)$ be satisfied. Let $A \in \mathcal{A}$ and $n \in \mathbb{N}$. Then there exists a solution $x$ of the boundary value problem $(\mathrm{E})_{n}^{1},(1.2)$ satisfying (2.8) and (2.9), where $\Lambda_{A}$ is defined by (2.10).

Proof. Let us put

$$
\begin{array}{r}
\Omega=\left\{(x, c):(x, c) \in C^{1}(J) \times \mathbb{R},\|x\|<\max \left\{\left|\Lambda_{A}\right|,|A|\right\}+1,\right. \\
\left.\left\|x^{\prime}\right\| \leq H^{-1}\left(Q_{A}\left(\Lambda_{A}\right)\right)+1,|c|<\max \left\{\left|\Lambda_{A}\right|,|A|\right\}+1\right\} .
\end{array}
$$

Then $\Omega$ is a bounded, open, and symmetric with respect to $(0,0)$ subset of the Banach space $C^{1}(J) \times \mathbb{R}$ with the norm $\|(x, c)\|_{*}=\|x\|+\left\|x^{\prime}\right\|+|c|$. Define the mapping $\mathcal{F}: \bar{\Omega} \times[0,1] \rightarrow C^{1}(J) \times \mathbb{R}$ by the equality

$$
\mathcal{F}(x, c, \lambda)=(c, c+\max \{x(t): t \in J\}-\lambda A) .
$$

Then $\mathcal{F}$ is a compact operator. Let $\mathcal{I}$ be the identity operator on $C^{1}(J) \times \mathbb{R}$ and let us define $\mathcal{G}: \bar{\Omega} \rightarrow C^{1}(J) \times \mathbb{R}$ by putting $\mathcal{G}=\mathcal{I}-\mathcal{F}(\cdot, \cdot, 0)$. Then

$$
\mathcal{G}(x, c)=(x-c,-\max \{x(t): t \in J\}) .
$$

Assume now that

$$
\mathcal{G}\left(-x_{0},-c_{0}\right)=\chi_{0} \mathcal{G}\left(x_{0}, c_{0}\right)
$$

for some $\left(x_{0}, c_{0}\right) \in \partial \Omega$ and $\chi_{0} \in[1, \infty)$. Then

$$
\left(-x_{0}+c_{0},-\max \left\{-x_{0}(t): t \in J\right\}\right)=\chi_{0}\left(x_{0}-c_{0},-\max \left\{x_{0}(t): t \in J\right\}\right),
$$


and, thus,

$$
\begin{gathered}
\left(x_{0}(t)-c_{0}\right)\left(1+\chi_{0}\right)=0 \quad \text { for } t \in J, \\
\max \left\{-x_{0}(t): t \in J\right\}=\chi_{0} \max \left\{x_{0}(t): t \in J\right\} .
\end{gathered}
$$

It follows from (2.13) that $x_{0}(t)=c_{0}$ for $t \in J$, and (2.14) then yields $\left(\chi_{0}+1\right) c_{0}=0$. Hence, $\left(x_{0}, c_{0}\right)=(0,0)$, contrary to the inclusion $\left(x_{0}, c_{0}\right) \in \partial \Omega$. Now Theorem 8.3 from [7] implies that

$$
D(\mathcal{I}-\mathcal{F}(\cdot, \cdot, 0), \Omega, 0) \neq 0,
$$

where " $D$ " stands for the Leray-Schauder degree.

If $\mathcal{F}\left(x_{*}, c_{*}, \lambda_{*}\right)=\left(x_{*}, c_{*}\right)$ for some $\left(x_{*}, c_{*}\right) \in \partial \Omega$ and $\lambda_{*} \in[0,1]$, then $x_{*}=c_{*}$ and $\max \left\{x_{*}(t): t \in J\right\}=\lambda_{*} A$. Hence, $c_{*}=\lambda_{*} A$ and, consequently, $\left|c_{*}\right| \leq|A|,\left\|x_{*}\right\| \leq|A|$, and $\left\|x^{\prime}\right\|=0$, which contradicts the relation $\left(x_{*}, c_{*}\right) \in \partial \Omega$. We have thus proved that

$$
\mathcal{F}(x, c, \lambda) \neq(x, c)
$$

for $(x, c) \in \partial \Omega$ and $\lambda \in[0,1]$ and, therefore, by virtue of the homotopy property and (2.15), we obtain

$$
D(\mathcal{I}-\mathcal{F}(\cdot, \cdot, 1), \Omega, 0)=D(\mathcal{I}-\mathcal{F}(\cdot, \cdot, 0), \Omega, 0) \neq 0 .
$$

Finally, let us define the operator $\mathcal{K}: \bar{\Omega} \times[0,1] \rightarrow C^{1}(J) \times \mathbb{R}$ by the relation

$$
\begin{array}{r}
\mathcal{K}(x, c, \lambda)=\left(c+\int_{0}^{t} g^{-1}\left(K+\lambda \int_{0}^{s} f_{n}\left(v, x(v), x^{\prime}(v)\right) d v\right) d s,\right. \\
c+\max \{x(t): t \in J\}-A)
\end{array}
$$

where $K=K(x, \lambda) \in \mathbb{R}$ is a solution of the equation

$$
p(X ; x, \lambda)=0
$$

with

$$
p(X ; x, \lambda)=\int_{0}^{T} g^{-1}\left(X+\lambda \int_{0}^{t} f_{n}\left(s, x(s), x^{\prime}(s)\right) d s\right) d t .
$$

Since $p(\cdot ; x, \lambda)$ is continuous and increasing on $\mathbb{R}$ and $\lim _{X \rightarrow \pm \infty} p(X ; x, \lambda)= \pm \infty$ by virtue of $\left(H_{1}\right)$, it follows that equation (2.17) has a unique solution for every $(x, \lambda) \in \bar{\Omega} \times[0,1]$. Assume that $(x, c)$ is a fixed point of the operator $\mathcal{K}(\cdot, \cdot, 1)$. Then

$$
x(t)=c+\int_{0}^{t} g^{-1}\left(K_{*}+\int_{0}^{s} f_{n}\left(v, x(v), x^{\prime}(v)\right) d v\right) d s, \quad t \in J
$$

and $\max \{x(t): t \in J\}=A$, where $K_{*}$ is the unique solution of equation $p(X ; x, 1)=0$. Hence, $x$ is a solution of the boundary value problem $(\mathrm{E})_{n}^{1},(1.2)$ and $c=x(0)$. We see that, to prove our lemma, it is sufficient to verify that

$$
D(\mathcal{I}-\mathcal{K}(\cdot, \cdot, 1), \Omega, 0) \neq 0 .
$$

Since

$$
\mathcal{K}(x, c, 0)=(c, c+\max \{x(t): t \in J\}-A)
$$


we have $\mathcal{K}(\cdot, \cdot, 0)=\mathcal{F}(\cdot, \cdot, 1)$, and then $(2.16)$ yields the inequality

$$
D(\mathcal{I}-\mathcal{K}(\cdot, \cdot, 0), \Omega, 0) \neq 0 .
$$

By the homotopy property, the fulfilment of (2.19) will be proved if we show that

(i) $\mathcal{K}$ is a compact operator and

(ii) $\mathcal{K}(x, c, \lambda) \neq(x, c)$ for $(x, c) \in \partial \Omega$ and $\lambda \in[0,1]$.

We first show that $\mathcal{K}$ is a continuous operator. Let $\left\{\left(x_{m}, c_{m}, \lambda_{m}\right)\right\} \subset \bar{\Omega} \times[0,1]$ be a convergent sequence and $\lim _{m \rightarrow \infty}\left(x_{m}, c_{m}, \lambda_{m}\right)=\left(x_{0}, c_{0}, \lambda_{0}\right)$. Let $K_{m}$ be the unique solution of equation $p\left(X ; x_{m}, \lambda_{m}\right)=0$. Since $f_{n} \in \operatorname{Car}\left(J \times \mathbb{R}^{2}\right)$, there exists $\gamma \in L_{1}(J)$ such that

$$
\begin{aligned}
\left|f_{n}(t, x, y)\right| \leq & \gamma(t) \quad \text { for a. e. } t \in J \text { and all }(x, y) \\
& \text { such that }|x| \leq \max \left\{\left|\Lambda_{A}\right|,|A|\right\}+1 \text { and }|y| \leq H^{-1}\left(Q_{A}\left(\Lambda_{A}\right)\right)+1 .
\end{aligned}
$$

Therefore,

$$
p\left(\|\gamma\|_{L} ; x_{m}, \lambda_{m}\right)=\int_{0}^{T} g^{-1}\left(\|\gamma\|_{L}+\lambda_{m} \int_{0}^{t} f_{n}\left(v, x_{m}(v), x_{m}^{\prime}(v)\right) d v\right) d t \geq 0
$$

for $m \in \mathbb{N}$.

Now from $p\left(0 ; x_{m}, \lambda_{m}\right) \leq 0$ for $m \in \mathbb{N}$ we deduce that $\left\{K_{m}\right\}$ is bounded and $0 \leq K_{m} \leq\|\gamma\|_{L}$. If $\left\{K_{m}\right\}$ is not convergent, there exist subsequences $\left\{k_{m}\right\}$ and $\left\{l_{m}\right\}$ of $\{m\}$ such that $\lim _{m \rightarrow \infty} K_{k_{m}}=V_{1}, \lim _{m \rightarrow \infty} K_{l_{m}}=V_{2}$ and $V_{1} \neq V_{2}$. Then $p\left(V_{j} ; x_{0}, \lambda_{0}\right)=0(j=1,2)$ by the Lebesgue dominated convergence theorem, contrary to the fact that $p\left(\cdot ; x_{0}, \lambda_{0}\right)$ increasing. Hence $\left\{K_{m}\right\}$ is convergent and the continuity of $\mathcal{K}$ follows again from the Lebesgue dominated convergence theorem. By (2.20) and the Bolzano-Weierstrass theorem we can verify that $\mathcal{K}(\bar{\Omega} \times[0,1])$ is relatively compact in $C^{1}(J) \times \mathbb{R}$. Hence, property (i) is valid.

Assume that $\mathcal{K}(\bar{x}, \bar{c}, \bar{\lambda})=(\bar{x}, \bar{c})$ for some $(\bar{x}, \bar{c}) \in \bar{\Omega}$ and $\bar{\lambda} \in[0,1]$. If $\bar{\lambda}=0$, then from the first part of our proof we have $\bar{x}=\bar{c}=A$, and so $(\bar{x}, \bar{c}) \notin \partial \Omega$. Let $\bar{\lambda} \in(0,1]$. Then it can be readily verified that $\bar{x}$ is a solution of the boundary value problem $(\mathrm{E})_{n}^{\bar{\lambda}},(1.2)$ and $\bar{c}=\bar{x}(0)$. Hence, by Lemma $2.2,\|\bar{x}\| \leq \max \left\{\left|\Lambda_{A}\right|,|A|\right\},\left\|\bar{x}^{\prime}\right\| \leq$ $H^{-1}\left(Q_{A}\left(\Lambda_{A}\right)\right)$ and, therefore,

$$
|\bar{c}| \leq \max \left\{\left|\Lambda_{A}\right|,|A|\right\} .
$$

We have proved that $(\bar{x}, \bar{c}) \notin \partial \Omega$, which shows that condition (ii) is satisfied.

Lemma 2.4. Let assumptions $\left(H_{1}\right)-\left(H_{4}\right)$ be satisfied and $A \in \mathcal{A}$. Let $x_{n}$ be a solution of the boundary value problem $(\mathrm{E})_{n}^{1},(1.2)$ and $x_{n}^{\prime}\left(\xi_{n}\right)=0$ for a (unique) $\xi \in(0, T)$. Then there exist constants $c_{1}, c_{2}$ such that

$$
0<c_{1} \leq \xi_{n} \leq c_{2}<T \quad \text { for } n \in \mathbb{N} .
$$


Proof. Assume that there is a subsequence $\left\{\xi_{k_{n}}\right\}$ of $\left\{\xi_{n}\right\}$ such that $\lim _{n \rightarrow \infty} \xi_{k_{n}}=0$. Then

$$
A-x_{k_{n}}(0)=x_{k_{n}}\left(\xi_{k_{n}}\right)-x_{k_{n}}(0)=x_{k_{n}}^{\prime}\left(\tau_{n}\right) \xi_{k_{n}},
$$

where $\tau_{n} \in\left(0, \xi_{k_{n}}\right)$. By Lemma 2.1, we have

$$
A-x_{k_{n}}(0) \geq \int_{0}^{T / 2} g^{-1}(a s) d s
$$

and, therefore,

$$
\lim _{n \rightarrow \infty} x_{k_{n}}^{\prime}\left(\tau_{n}\right)=\left(A-x_{k_{n}}(0)\right) / \xi_{k_{n}}=\infty,
$$

contrary to the inequality $\left\|x_{n}^{\prime}\right\| \leq H^{-1}\left(Q_{A}\left(\Lambda_{A}\right)\right)$ for $n \in \mathbb{N}$ (see Lemma 2.2). Hence $\xi_{n} \geq c_{1}$ for $n \in \mathbb{N}$ with a positive constant $c_{1}$. The inequalities $\xi_{n} \leq c_{2}<T, n \in \mathbb{N}$, with a constant $c_{2}$ can be proved analogously.

Lemma 2.5. Let assumptions $\left(H_{1}\right)-\left(H_{4}\right)$ be satisfied and $A \in \mathcal{A}$. Let $x_{n}$ be a solution of the boundary value problem $(\mathrm{E})_{n}^{1},(1.2)$. Then the sequence

$$
\left\{f_{n}\left(t, x_{n}(t), x_{n}^{\prime}(t)\right)\right\} \subset L_{1}(J)
$$

is uniformly absolutely continuous (UAC) on $J$, that is, for every $\varepsilon>0$, there exists a $\delta>0$ such that

$$
\int_{\mathcal{M}}\left|f_{n}\left(t, x_{n}(t), x_{n}^{\prime}(t)\right)\right| d t<\varepsilon, \quad n \in \mathbb{N}
$$

whenever $\mathcal{M} \subset J$ is measurable and $\mu(\mathcal{M})<\delta$.

Proof. By Lemma 2.2,

$$
\left\|x_{n}\right\| \leq \max \left\{\left|\Lambda_{A}\right|,|A|\right\}, \quad\left\|x_{n}^{\prime}\right\| \leq H^{-1}\left(Q_{A}\left(\Lambda_{A}\right)\right) \quad \text { for } n \in \mathbb{N},
$$

where the constant $\Lambda_{A}$ is defined by (2.10), and then (see (2.1))

$$
\begin{aligned}
\left|f_{n}\left(t, x_{n}(t), x_{n}^{\prime}(t)\right)\right| \leq & \max \left\{q(u):|u| \leq \max \left\{\left|\Lambda_{A}\right|,|A|\right\}\right\} \times \\
& \times\left(\max \left\{\omega_{* 1}(u): 0 \leq u \leq H^{-1}\left(Q_{A}\left(\Lambda_{A}\right)\right)\right\}+\omega_{2}\left(\left|x_{n}^{\prime}(t)\right|\right)\right)
\end{aligned}
$$

for a. e. $t \in J$ and every $n \in \mathbb{N}$. In addition, $x^{\prime}\left(\xi_{n}\right)=0$ for a unique $\xi_{n} \in(0, T)$,

$$
\begin{array}{cc}
x_{n}^{\prime}(t) \geq g^{-1}\left(a\left(\xi_{n}-t\right)\right) & \text { for } t \in\left[0, \xi_{n}\right], \\
\left|x_{n}^{\prime}(t)\right| \geq g^{-1}\left(a\left(t-\xi_{n}\right)\right) & \text { for } t \in\left[\xi_{n}, T\right]
\end{array}
$$

for $n \in \mathbb{N}$ by Lemma 2.1, and (2.21) is true with constants $c_{1}, c_{2}$. Now (2.23) yields that $\left\{f_{n}\left(t, x_{n}(t), x_{n}^{\prime}(t)\right)\right\}$ is $\mathrm{UAC}$ on $J$ if $\left\{\omega_{2}\left(\left|x_{n}^{\prime}(t)\right|\right)\right\}$ is UAC on $J$.

Due to the structure of the measurable sets in $\mathbb{R}$, it follows that $\left\{\omega_{2}\left(\left|x_{n}^{\prime}(t)\right|\right)\right\}$ is UAC on $J$ if and only if for every $\varepsilon>0$ there exists $\delta>0$ such that for any at most countable set $\left\{\left(a_{j}, b_{j}\right)\right\}_{j \in \mathbb{J}}$ of the mutually disjoint intervals $\left(a_{j}, b_{j}\right) \subset J, \sum_{j \in \mathbb{J}}\left(b_{j}-\right.$ $\left.a_{j}\right)<\delta$, we have

$$
\sum_{j \in \mathbb{J}} \int_{a_{j}}^{b_{j}} \omega_{2}\left(\left|x_{n}^{\prime}(t)\right|\right) d t<\varepsilon, \quad n \in \mathbb{N} .
$$


For this reason, let $\left\{\left(a_{j}, b_{j}\right)\right\}_{j \in \mathrm{J}}$ be an at most countable set of mutually disjoint intervals $\left(a_{j}, b_{j}\right) \subset J$. Let us put

$$
\mathbb{J}_{n}^{1}=\left\{j: j \in \mathbb{J},\left(a_{j}, b_{j}\right) \subset\left(0, \xi_{n}\right)\right\}
$$

and

$$
\mathbb{J}_{n}^{2}=\left\{j: j \in \mathbb{J},\left(a_{j}, b_{j}\right) \subset\left(\xi_{n}, T\right)\right\} .
$$

Then, for $j \in \mathbb{J}_{n}^{1}$ and $i \in \mathbb{J}_{n}^{2}$, in view of (2.24) and (2.25), we have

$$
\int_{a_{j}}^{b_{j}} \omega_{2}\left(\left|x_{n}^{\prime}(t)\right|\right) d t \leq \int_{a_{j}}^{b_{j}} \omega_{2}\left(g^{-1}\left(a\left(\xi_{n}-t\right)\right)\right) d t=\frac{1}{a} \int_{a\left(\xi_{n}-b_{j}\right)}^{a\left(\xi_{n}-a_{j}\right)} \omega_{2}\left(g^{-1}(s)\right) d s
$$

and

$$
\int_{a_{i}}^{b_{i}} \omega_{2}\left(\left|x_{n}^{\prime}(t)\right|\right) d t \leq \int_{a_{i}}^{b_{i}} \omega_{2}\left(g^{-1}\left(a\left(t-\xi_{n}\right)\right)\right) d t=\frac{1}{a} \int_{a\left(a_{i}-\xi_{n}\right)}^{a\left(b_{i}-\xi_{n}\right)} \omega_{2}\left(g^{-1}(s)\right) d s .
$$

If $\mathbb{J}_{n}^{1} \cup \mathbb{J}_{n}^{2} \neq \mathbb{J}$ and $\left\{j_{0}\right\}=\mathbb{J} \backslash\left(\mathbb{J}_{n}^{1} \cup \mathbb{J}_{n}^{2}\right)$, then

$$
\begin{aligned}
\int_{a_{j_{0}}}^{b_{j_{0}}} \omega_{2}\left(\left|x_{n}^{\prime}(t)\right|\right) d t & \leq \int_{a_{j_{0}}}^{\xi_{n}} \omega_{2}\left(g^{-1}\left(a\left(\xi_{n}-t\right)\right)\right) d t+\int_{\xi_{n}}^{b_{j_{0}}} \omega_{2}\left(g^{-1}\left(a\left(t-\xi_{n}\right)\right)\right) d t \\
& =\frac{1}{a}\left[\int_{0}^{a\left(\xi_{n}-a_{j_{0}}\right)} \omega_{2}\left(g^{-1}(s)\right) d s+\int_{0}^{a\left(b_{j_{0}}-\xi_{n}\right)} \omega_{2}\left(g^{-1}(s)\right) d s\right] .
\end{aligned}
$$

Let us set

$$
\Delta_{n}^{1}=\bigcup_{j \in \mathbb{J}_{n}^{1}}\left(a\left(\xi_{n}-b_{j}\right), a\left(\xi_{n}-a_{j}\right)\right)+\mathcal{E}_{n}^{1}
$$

and

$$
\Delta_{n}^{2}=\bigcup_{j \in \mathbb{J}_{n}^{2}}\left(a\left(a_{j}-\xi_{n}\right), a\left(b_{j}-\xi_{n}\right)\right)+\mathcal{E}_{n}^{2}
$$

where

$$
\mathcal{E}_{n}^{1}= \begin{cases}\varnothing & \text { if } \mathbb{J}=\mathbb{J}_{n}^{1} \cup \mathbb{J}_{n}^{2} \\ \left(0, a\left(\xi_{n}-a_{j_{0}}\right)\right) & \text { if }\left\{j_{0}\right\}=\mathbb{J} \backslash\left(\mathbb{J}_{n}^{1} \cup \mathbb{J}_{n}^{2}\right)\end{cases}
$$

and

$$
\mathcal{E}_{n}^{2}= \begin{cases}\varnothing & \text { if } \mathbb{J}=\mathbb{J}_{n}^{1} \cup \mathbb{J}_{n}^{2} \\ \left(0, a\left(b_{j_{0}}-\xi_{n}\right)\right) & \text { if }\left\{j_{0}\right\}=\mathbb{J} \backslash\left(\mathbb{J}_{n}^{1} \cup \mathbb{J}_{n}^{2}\right) .\end{cases}
$$

Then

$$
\mu\left(\Delta_{n}^{i}\right) \leq a \sum_{j \in \mathbb{J}}\left(b_{j}-a_{j}\right), \quad i=1,2
$$


and

$$
\sum_{j \in \mathbb{J}} \int_{a_{j}}^{b_{j}} \omega_{2}\left(\left|x_{n}^{\prime}(t)\right|\right) d t \leq \frac{1}{a}\left[\int_{\Delta_{n}^{1}} \omega_{2}\left(g^{-1}(t)\right) d t+\int_{\Delta_{n}^{2}} \omega_{2}\left(g^{-1}(t)\right) d t\right] .
$$

Since $\omega_{2}\left(g^{-1}\right) \in L_{\text {loc }}([0, \infty))$, we conclude from (2.26) and (2.27) that the sequence $\left\{\omega_{2}\left(\left|x_{n}^{\prime}(t)\right|\right)\right\}$ is UAC on $J$.

\section{EXISTENCE RESULT AND AN EXAMPLE}

Theorem 3.1. Let assumptions $\left(H_{1}\right)-\left(H_{4}\right)$ be satisfied. Then boundary value problem (1.1), (1.2) has a solution for every $A \in \mathcal{A}$.

Proof. Let us fix $A \in \mathcal{A}$. By Lemmas 2.1-2.4, there exists a solution $x_{n}$ of the boundary value problem $(\mathrm{E})_{n}^{1},(1.2)$ and inequalities (2.21)-(2.25) are satisfied, where $\Lambda_{A}$ is defined by (2.10), $\xi_{n} \in(0, T)$ is the unique zero of $x_{n}^{\prime}$ and $c_{1}, c_{2}$ are constants. In addition, by Lemma 2.5, the sequence $\left\{f_{n}\left(t, x_{n}(t), x_{n}^{\prime}(t)\right)\right\}$ is UAC on $J$, which implies that the sequence

$$
\left\{g\left(x_{n}^{\prime}(t)\right)\right\}=\left\{\int_{\xi_{n}}^{t} f_{n}\left(s, x_{n}(s), x_{n}^{\prime}(s)\right) d s\right\}
$$

is equicontinuous on $J$. Then, in view of the relation

$$
\left|x_{n}^{\prime}\left(t_{2}\right)-x_{n}^{\prime}\left(t_{1}\right)\right|=\left|g^{-1}\left(g\left(x_{n}^{\prime}\left(t_{2}\right)\right)\right)-g^{-1}\left(g\left(x_{n}^{\prime}\left(t_{1}\right)\right)\right)\right|, \quad t_{1}, t_{2} \in J, n \in \mathbb{N} ;
$$

and the fact that $g^{-1}$ is continuous and increasing on $\mathbb{R}$, we conclude that $\left\{x_{n}^{\prime}(t)\right\}$ is equicontinuous on $J$. Hence, $\left\{x_{n}\right\}$ is bounded in $C^{1}(J),\left\{x_{n}^{\prime}(t)\right\}$ is equicontinuous on $J$ and since $\left\{\xi_{n}\right\}$ is bounded, using the Arzelà-Ascoli theorem and the BolzanoWeierstrass theorem, we can assume without loss of generality that $\left\{x_{n}\right\}$ is convergent in $C^{1}(J)$ and $\left\{\xi_{n}\right\}$ is convergent in $\mathbb{R}$.

Let $\lim _{n \rightarrow \infty} x_{n}=x$ and $\lim _{n \rightarrow \infty} \xi_{n}=\xi$. Then $x \in C^{1}(J), x(0)=x(T), \max \{x(t)$ : $t \in J\}=A, 0<c_{1} \leq \xi \leq c_{2}<T$, and

$$
x^{\prime}(t) \geq g^{-1}(a(\xi-t)) \text { for } t \in[0, \xi], \quad\left|x^{\prime}(t)\right| \geq g^{-1}(a(t-\xi)) \text { for } t \in[\xi, T] .
$$

Therefore,

$$
\lim _{n \rightarrow \infty} f_{n}\left(t, x_{n}(t), x_{n}^{\prime}(t)\right)=f\left(t, x(t), x^{\prime}(t)\right) \quad \text { for a. e. } t \in J,
$$

Thus, $f\left(t, x(t), x^{\prime}(t)\right) \in L_{1}(J)$ and

$$
\lim _{n \rightarrow \infty} \int_{0}^{t} f_{n}\left(s, x_{n}(s), x_{n}^{\prime}(s)\right) d s=\int_{0}^{t} f\left(s, x(s), x^{\prime}(s)\right) d s, \quad t \in J,
$$

by the Vitali convergence theorem. Passing to the limit as $n \rightarrow \infty$ in the equalities

$$
g\left(x_{n}^{\prime}(t)\right)=\int_{\xi_{n}}^{t} f_{n}\left(s, x_{n}(s), x_{n}^{\prime}(s)\right) d s, \quad t \in J, n \in \mathbb{N}
$$


we get

$$
g\left(x^{\prime}(t)\right)=\int_{\xi}^{t} f\left(s, x(s), x^{\prime}(s)\right) d s, \quad t \in J .
$$

Hence, $g\left(x^{\prime}\right) \in A C(J)$ and $x$ is a solution of boundary value problem (1.1), (1.2).

Example 3.2. Consider the differential equation

$$
\left(\left|x^{\prime}(t)\right|^{p} \operatorname{sgn} x^{\prime}(t)\right)^{\prime}=-(2+\sin (t x(t))+|x(t)|)\left(\left|x^{\prime}(t)\right|^{\alpha}+\frac{1}{\left|x^{\prime}(t)\right|^{\beta}}\right)
$$

where $p>1,0 \leq \alpha<p-1$ and $0<\beta<p$.

Let us set

$$
f(t, x, y)=-(2+\sin (t x)+|x|)\left(|y|^{\alpha}+\frac{1}{|y|^{\beta}}\right)
$$

for $(t, x, y) \in J \times \mathbb{R} \times(\mathbb{R} \backslash\{0\})$. Then $\left(H_{1}\right)$ is satisfied with $g(u)=|u|^{p} \operatorname{sgn} u$, and $f$ satisfies $\left(H_{2}\right)$ and $\left(H_{3}\right)$ with $a=1, q(x)=3+|x|, \omega_{1}(u)=u^{\alpha}$ and $\omega_{2}(u)=1 / u^{\beta}$. Therefore,

$$
\omega_{* 1}(u)= \begin{cases}1 & \text { for } 0 \leq u \leq 1 \\ u^{\alpha} & \text { for } u>1\end{cases}
$$

and

$$
\begin{gathered}
Q_{A}(u)=\int_{u}^{A} q(s) d s=\int_{u}^{A}(3+|s|) d s \geq 3(A-u), \\
Q_{A}(u)=\int_{u}^{A}(3+|s|) d s \leq(3+\max \{|A|,|u|\})(A-u)
\end{gathered}
$$

for $u \leq A$. Since

$$
\begin{aligned}
H(u) & =\int_{0}^{g(u)} \frac{g^{-1}(s)}{\omega_{* 1}\left(g^{-1}(s)\right)+\omega_{2}\left(g^{-1}(s)\right)} d s=\int_{0}^{u^{p}} \frac{s^{1 / p}}{1+s^{-\beta / p}} d s \\
& \leq \int_{0}^{u^{p}} s^{(1+\beta) / p} d s=\frac{p}{1+\beta+p} u^{1+\beta+p}
\end{aligned}
$$

for $u \in[0,1]$, we have that

$$
H^{-1}(u) \geq\left(\frac{1+\beta+p}{p} u\right)^{1 /(1+\beta+p)}
$$

for $u \in[0, H(1)]$ and, hence (see (3.2)),

$$
\int_{A-\varepsilon}^{A} \frac{1}{H^{-1}\left(Q_{A}(s)\right)} d s \leq \int_{A-\varepsilon}^{A}\left(\frac{3(1+\beta+p)}{p}(A-s)\right)^{-1 /(1+\beta+p)} d s<\infty
$$

for $\varepsilon=\min \left\{1, A-Q_{A}^{-1}(H(1))\right\}$ because $1 /(1+\beta+p)<1$. This shows that $\left(H_{4}\right)$ is satisfied. 
We are going to verify that $\mathcal{A}=\mathbb{R}$, where $\mathcal{A}$ is given by (2.7). For $u \geq 1$, we have

$$
\begin{aligned}
H(u) & =H(1)+\int_{1}^{u^{p}} \frac{s^{1 / p}}{s^{\alpha / p}+s^{-\beta / p}} d s=H(1)+\int_{1}^{u^{p}} \frac{s^{(1+\beta) / p}}{1+s^{(\alpha+\beta) / p}} d s \\
& \geq H(1)+\frac{1}{2} \int_{1}^{u^{p}} s^{(1-\alpha) / p} d s=H(1)+\frac{p}{2(1+p-\alpha)}\left(u^{1+p-\alpha}-1\right)
\end{aligned}
$$

and, consequently,

$$
H^{-1}(u) \leq\left(\frac{2(1+p-\alpha)}{p}(u-H(1))+1\right)^{1 /(1+p-\alpha)}
$$

for $u \in[H(1), \infty)$. Hence, we have (see (3.3))

$$
\begin{aligned}
\int_{u}^{A} & \frac{1}{H^{-1}\left(Q_{A}(s)\right)} d s=\int_{Q_{A}^{-1}(H(1))}^{A} \frac{1}{H^{-1}\left(Q_{A}(s)\right)} d s+\int_{u}^{Q_{A}^{-1}(H(1))} \frac{1}{H^{-1}\left(Q_{A}(s)\right)} d s \\
> & \int_{u}^{Q_{A}^{-1}(H(1))}\left[\frac{2(1+p-\alpha)}{p}((3+\max \{|A|,|s|\})(A-s)-H(1))+1\right]^{-\frac{1}{1+p-\alpha}} d s
\end{aligned}
$$

for $u<Q_{A}^{-1}(H(1))$. Since $2 /(1+p-\alpha)<1$, we see that

$$
\lim _{u \rightarrow-\infty} \int_{u}^{A} \frac{1}{H^{-1}\left(Q_{A}(s)\right)} d s=\infty
$$

for every $A \in \mathbb{R}$ and, hence, we have proved that $\mathcal{A}=\mathbb{R}$. Applying Theorem 3.1, we conclude that the boundary value problem (3.1), (1.2) has a solution for all $A \in \mathbb{R}$.

\section{REFERENCES}

[1] Agarwal, R. P., O'Regan, D., and StaněK, S.: Singular Lidstone boundary value problem with given maximal values for solutions, to appear.

[2] Agarwal, R. P., O'Regan, D., And StanĚK, S.: Solvability of singular Dirichlet boundary value problems with given maximal values for positive solutions, to appear.

[3] Bartle, R. G.: A Modern Theory of Integration, Amer. Math. Soc., Providence, R. I., 2001.

[4] Brykalov, S. A.: Solutions with a prescribed minimum and maximum, Differents. Uravn., 29 (1993), 638-642 (in Russian). English transl. in Differential Equations, 29 (1993), 802-805.

[5] Brykalov, S. A.: Solvability of problems with monotone boundary conditions, Differents. Uravn., 29 (1993), 744-750 (in Russian). English transl. in Differential Equations, 29 (1993), 633-639.

[6] BRYKalov, S. A.: Multiplicity results for problems with uniform norms in boundary conditions, Nonlinear Anal., 30 (1997), 4781-4788.

[7] Deimling, K: Nonlinear Functional Analysis, Springer-Verlag, Berlin, Heidelberg, etc., 1985.

[8] FečKAn, M.: The interaction of linear boundary value and nonlinear functional conditions, Ann. Polon. Math., 58 (1993), 299-310.

[9] HaKL, R., KiguRADZe, I., AND PŮŽA, B.: Upper and lower solutions to boundary value problems for functional-differential equations and theorems on functional-differential inequalities, Georgian Math. J., 7 (2000), 489-512.

[10] НАŠÁк, A., Pirč, V., AND StAnĚK, S.: On boundary value problems for third-order functional differential equations, Nonlinear Anal., 42 (2000), 1077-1089. 
[11] Hewitt, E. And Stromberg, S.: Real and Abstract Analysis, Springer-Verlag, Berlin, Heidelberg, etc., 1965.

[12] Kiguradze, I. And PŮža, B.: On boundary value problems for functional differential equations, Mem. Differential Equations Math. Phys., 12 (1997), 106-113.

[13] Kiguradze, I. AND PŮžA, B.: On the solvability of nonlinear boundary value problems for functional differential equations, Georgian Math. J., 5 (1998), 251-262.

[14] Kiguradze, I. T. and Shekhter, B. L.: Singular boundary value problems for second order ordinary differential equations, In: "Current Problems in Math.: Newest Results," Moscow, 30, 105-201 (in Russian). English transl. in J. Soviet Math., 43 (1988), 2340-2417.

[15] Lomtatidze, A. and Malaguti, L.: On a nonlocal boundary value problem for second order nonlinear singular differential equations, Georgian Math. J., 7 (2000),133-154.

[16] Lepin, A. YA. And Myshis, A. D.: General nonlocal nonlinear boundary value problem for differential equation of 3rd order, Nonlinear Anal., 28 (1997), 1533-1543.

[17] STANĚK, S.: Multiplicity results for second order nonlinear problems with maximum and minimum, Math. Nachr., 192 (1998), 225-237.

[18] STANĚK, S.: Existence and multiplicity of solutions for functional boundary value problems, Nonlinear Anal., 47 (2001), 3925-3936.

[19] STANĚK, S.: Two functional boundary value problems with singularities in phase variables, Nonlinear Oscillations, 5 (2002), 387-415.

[20] STANĚK, S.: On nonlocal boundary value problem with singularities in phase variables, submitted.

\section{Author's Address}

\section{Svatoslav Staněk:}

Department of Mathematical Analysis, Faculty of Science, Palacký University, Tomkova 40, 77900 Olomouc, Czech Republic

E-mail address: stanek@risc.upol.cz 